Journal of Animal and Veterinary Advances 11 (13): 2352-2355, 2012

ISSN: $1680-5593$

(C) Medwell Journals, 2012

\title{
Expression of MyD88 Protein in Buffalo Tissues
}

Yongchang Hao, Hanwei Jiao, Li Du, Donglin Zhang, Ying Cheng, Ming Lei,

Hui Rong, Jianing Zhang, Xiaoxiao Jia and Fengyang Wang

Hainan Key Laboratory of Tropical Animal Reproduction and Breeding and

Epidemic Disease Research (Construction Period), Animal Genetic Engineering Key Lab of Haikou, College of Agriculture, Hainan University, 570228 Haikou, People's Republic of China

\begin{abstract}
Brucellosis is an extremely important disease affecting the health of human and a number of animal species (including buffalo) around the world and Brucellae sp. is the causative agent of brucellosis. The innate immune system is the first line of defense mechanisms that protect hosts from invading Brucella. As an important innate immunity molecule, MyD88 is critical for TLR-mediated activation of the transcription factor NF- $\mathrm{kB}$ and the induction of proinflammatory cytokines. To analyze the expression of MyD88 protein and provide new alternatives for elucidating the molecular mechanism of Brucella infection in buffalo, Western blot were performed to assess the expression of MyD88 protein in heart, liver, spleen, lung, kidney and small intestine, immunohistochemistry techniques were applied for evaluating the distribution of MyD88 protein. The results indicated that MyD88 protein are ubiquitously present in all buffalo tissues examined with relatively high levels in lung and relatively low levels in heart and small intestine and the subcellular localization of MyD88 is predominantly cytoplasm and the tissue specificity were observed. These data provide important anatomical information for studying the role of MyD88 played in Brucella infection in the different tissue of buffalo.
\end{abstract}

Key words: Buffalo, MyD88, Western blot, immunohistochemistry, tissue, expression

\section{INTRODUCTION}

Brucellosis is an extremely important disease affecting the health of human and a number of animal species (including buffalo) around the world and as facultative intracellular gram-negative coccobacilli, Brucella organisms are causative agents of brucellosis (Corbel, 1997; Hirsh et al., 2004).

The innate immune system is the first line of defense mechanisms that protect hosts from invading Brucella. As transmembrane receptors that sense lipids, lipoproteins, proteins and nucleic acids, Toll-Like Receptors (TLRs) upon activation by microbial products, transduce signals via a common adaptor molecule, Myeloid Differentiation factor 88 (MyD88), consequently leading to host cell activation. Activation of MyD88-dependent pathways results in NF- $\mathrm{kB}$ translocation to the nucleus and the induction of proinflammatory cytokines (Adachi et al., 1998; Byrd-Leifer et al., 2001; De Almeida et al., 2011; Macedo et al., 2008; Takeuchi et al., 2000).
Although, the findings on the expression of MyD88 protein in different tissues are able to provide important anatomical clues for evaluating the role of MyD88 played in the interaction between Brucella and target cells in the different tissue of animal, there are few reports about its expression level analysis in normal tissues of animal especially in buffalo. In this study to investigate the expression of MyD88 protein in the tissues of buffalo, Western blot were performed to assess the expression of MyD88 protein in heart, liver, spleen, lung, kidney and small intestine, respectively and immunohistochemistry techniques were applied for evaluating the distribution of MyD88 protein. The findings suggest that MyD88 protein are ubiquitously present in heart, liver, spleen, lung, kidney and small intestine of buffalo and the protein expression level in lung is the highest and relatively low levels in heart and small intestine; the subcellular localization of MyD88 is predominantly cytoplasm and the tissue specificity were observed which provide important anatomical information for elucidating the role of MyD88 played in Brucella infection in buffalo.

Corresponding Author: Fengyang Wang, Hainan Key Laboratory of Tropical Animal Reproduction and Breeding and Epidemic Disease Research (Construction Period), Animal Genetic Engineering Key Lab of Haikou, College of Agriculture, Hainan University, 570228 Haikou, People's Republic of China 


\section{MATERIALS AND METHODS}

Buffalo tissues: Adult Xinglong buffalo, a local buffalo breed were sacrificed and heart, liver, spleen, lung, kidney and intestine tissue were obtained immediately. The tissues used for Western blot were initially immersed in liquid nitrogen and preserved at $-70^{\circ} \mathrm{C}$. The tissues used for immunohistology analysis were washed with $\mathrm{NaCl}$ $(0.9 \%)$ and fixed with paraformaldehyde (4\%). All animal experimental protocols complied with national legislation and company policy on the care and use of animals according to the related code of practice.

Western blot: After about $0.1 \mathrm{~g}$ frozen tissues treated with liquid nitrogen were ground into powder, $1 \mathrm{~mL}$ tissue protein extract reagent (Biyuntian, China) and $10 \mu \mathrm{L}$ Phenylmethanesulfonyl Fluoride (PMSF) mixture were added and the powder were ground into liquid. After centrifugal $\left(15000 \mathrm{r} \mathrm{min}^{-1}\right)$ for $10 \mathrm{~min}$ at $4^{\circ} \mathrm{C}$, the supernatant were obtained and Bicinchoninic Acid (BCA) method were used to determine the protein concentrations.

Equal amounts of total protein $(50 \mu \mathrm{g})$ were separated by $10 \%$ Sodium Dodecyl Sulfate-polyacrylamide Gel Electrophoresis (SDS-PAGE) and electrophoretically transferred onto Polyvinylidene Fluoride (PVDF) membrane. Non-specific-binding sites were blocked with $5 \%$ BSA for $1 \mathrm{~h}$ at $37^{\circ} \mathrm{C}$, the membrane was incubated with primary antibodies at $4^{\circ} \mathrm{C}$ overnight, the primary antibodies were goat polyclonal anti-bovine MyD88 (1:1000 dilution) (Abcam, USA), Glyceraldehyde-3Phosphate Sehydrogenase (GAPDH) rabbit $\mathrm{mAB}$ (1:2000 dilution) (Millipore, USA), respectively. The secondary antibodies were Horseradish Peroxidase (HRP) labeled rabbit anti-goat $\operatorname{IgG}$ (1:4000 dilution) (Santa Cruz Biotechnology, USA) and HRP labeled goat anti-rabbit IgG (1:4000 dilution). After incubation with the secondary antibodies for $1 \mathrm{~h}$ at $37^{\circ} \mathrm{C}$, the membranes were developed with Enhanced Chemiluminescent Method (ECM) kit (Boster, China) to visualize and the proteins were detected by charge-coupled device cameras. Image J Software was used to quantify the Western blots for protein expression.

Immunohistology: Immunohistology were performed as follows: briefly, tissues were fixed into $4 \%$ formalin for $24 \mathrm{~h}$ at room temperature. after dehydration, transparent, paraffin embedding, $5 \mu \mathrm{m}$ sections were cut. After removing paraffin in several changes of xylene, the slides were hydrated through a series of graded alcohols to water and incubated with 3\% hydrogen peroxide for
$10 \mathrm{~min}$. The tissue sections were treated with goat polyclonal anti-bovine MyD88 antibody (1:100 dilution) (Abcam, USA) and incubated $1 \mathrm{~h}$ at $37^{\circ} \mathrm{C}$. After washing with PBS 3 times ( $5 \mathrm{~min} /$ time), polymer helper (Zhongshan Goldenbridge, China) was added to sections and incubated for $15 \mathrm{~min}$ at $37^{\circ} \mathrm{C}$ followed by washing with PBS 3 times ( $2 \mathrm{~min} /$ time $)$.

Poly-HRP anti-Goat IgG (Zhongshan Goldenbridge, China) was added to sections and incubated for $15 \mathrm{~min}$ at $37^{\circ} \mathrm{C}$ and followed by washing with Phosphate Buffer Saline (PBS) 3 times ( $2 \mathrm{~min} / \mathrm{time}$ ). Color was developed using a color developing kit (Boster, China). Slides were counterstained with hematoxylin (Beyotime, China). For negative control, normal goat serum or PBS alone were used in place of MyD88 antibody. The counts were observed under microscope made in five fields in two sections from each buffalo in the study.

\section{RESULTS AND DISCUSSION}

TLRs are one-pass transmembrane receptors containing extracellular leucine-rich repeat domains and an intracellular Toll/Interleukin-Receptor homology (TIR) domain. Upon activation, the TIR domain can recruit MyD88, one of several adaptor molecules that act directly downstream of TLRs. MyD88 is critical for TLR-mediated activation of the transcription factor NF- $\mathrm{kB}$ and thus for the induction of proinfiammatory cytokines such as Tumor Necrosis Factor $\alpha$ (TNF $\alpha$ ) (Sun and Ding, 2006; Takeda et al., 2003; Yamamoto et al., 2004; Weiss et al., 2005).

Previous studies in the laboratory indicated that buffalo MyD88 cDNA contained a 891 bp Open Reading Frame (ORF), encoding 296 amino acids (Zhang et al., 2011). In this study to further determine the expression level of MyD88 protein in all buffalo tissues examined and provide important anatomical information on the role of MyD88 in the interaction between Brucella and target cells in the different buffalo tissue, Western blot were performed, the results indicated hat MyD88 protein are ubiquitously present in all buffalo tissues examined with relatively high levels in lung and relatively low levels in heart and small intestine.

These data suggested that it is possible that MyD88 may play more important function in lung than that in other tissues examined. MyD88 protein levels in heart, liver, spleen, lung, kidney and small intestine were different and which likely to be correlated with its role in the interaction between Brucella and target cells in the different tissue of buffalo (Fig. 1). 


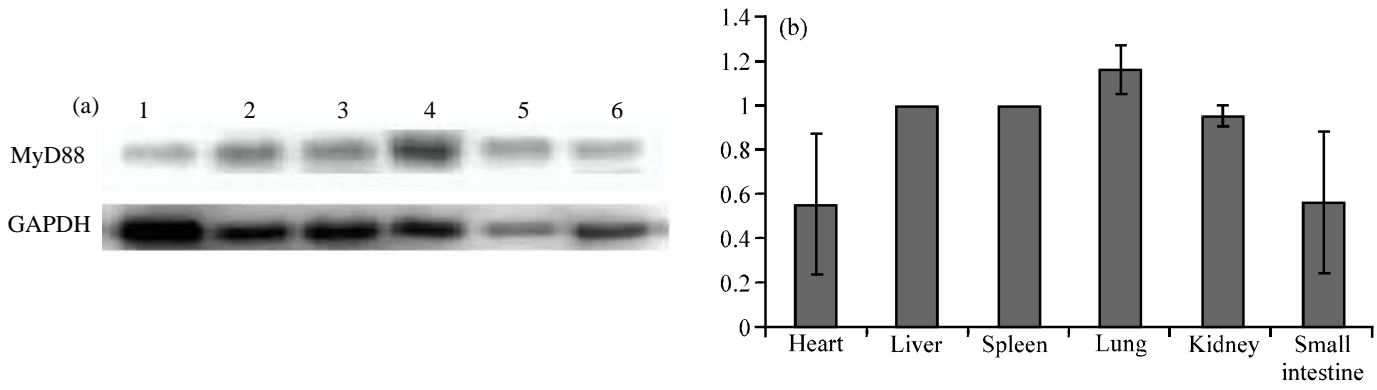

Fig. 1: a) Western blot analysis of MyD88 protein expression in buffalo: 1) Heart; 2) Liver; 3) Spleen; 4) Lung; 5) Kidney and 6) Small intestine tissues; b) The quantification of the Western blots for MyD88 protein expression with Image $J(n=3)$

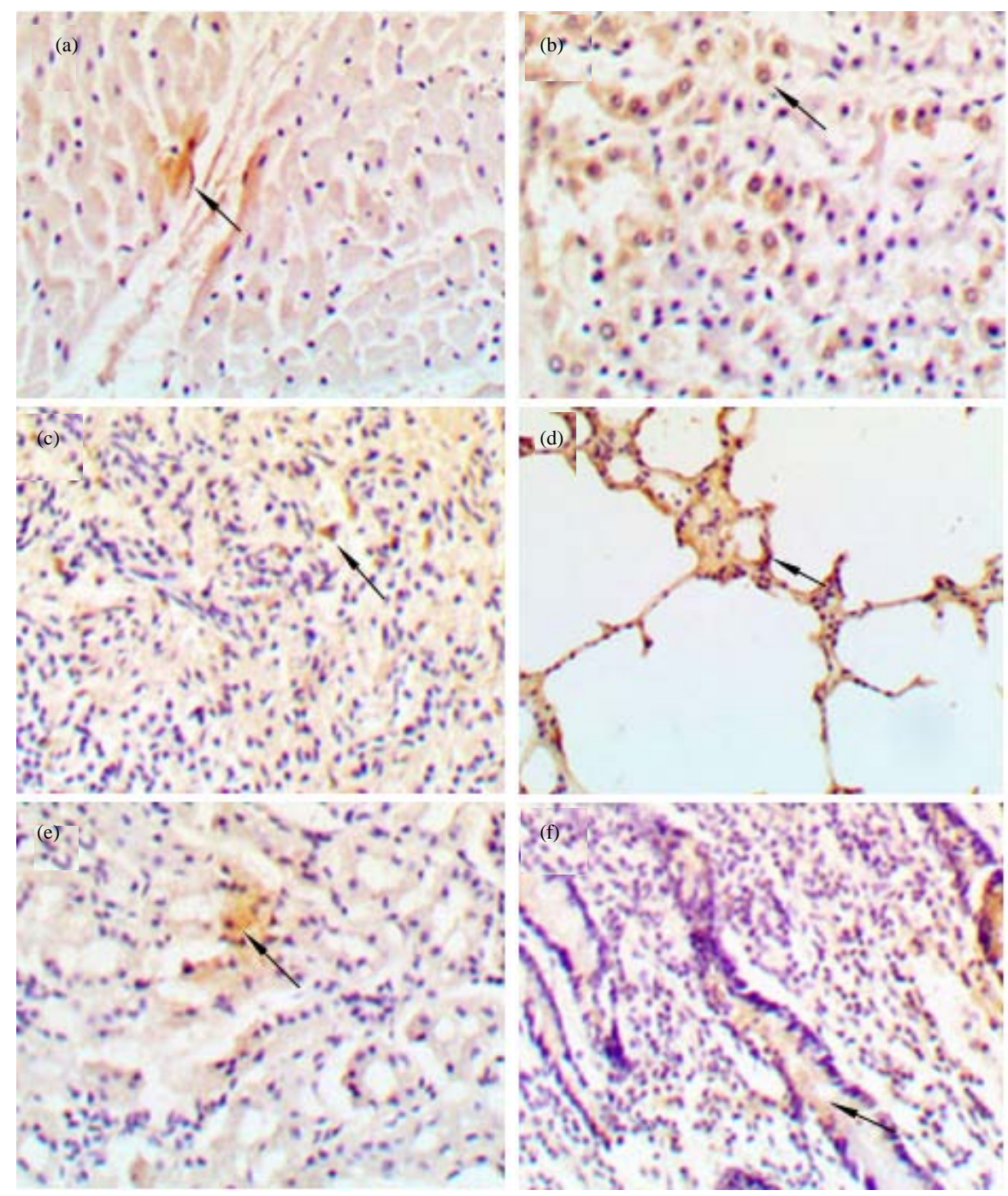

Fig. 2: Distribution of MyD88 protein in the different tissue cells of buffalo. Immunohistochemiistry detection of MyD88 in different tissues of buffalo: a) Heart; b) Liver; c) Spleen; d) Lung; e) Kidney; f) Small intestine 


\section{CONCLUSION}

The results of immunohistology test with goat polyclonal anti-bovine MyD88 antibody indicated that MyD88 protein are ubiquitously present in heart, liver, spleen, lung, kidney and small intestine and mainly exists in the cytoplasm and the weak positive signals were observed in heart, kidney and small intestine and strong positive signals were observed in spleen, lung and liver (Fig. 2).

The findings provide important anatomical information for studying the role of MyD88 played in Brucella infection in the different tissue of buffalo. Further studies are likely to include investigating the interaction of MyD88 in buffalo tissues with important Brucella bacterial proteins.

\section{ACKNOWLEDGEMENT}

This study was financially supported by the Major National Science and Technology Specific Projects (2009ZX08007-009B) and the National High Technology Research and Development Program of China (2011AA100302). Yongchang Hao, Hanwei Jiao, Li Du. The researcher contributed equally to this study.

\section{REFERENCES}

Adachi, O., T. Kawai, K. Takeda, M. Matsumoto and H. Tsutsui et al., 1998. Targeted disruption of the MyD88 gene results in loss of IL-1- and IL-18mediated function. Immunity, 9: 143-150.

Byrd-Leifer, C.A., E.F. Block, K. Takeda, S. Akira and A. Ding, 2001. The role of MyD88 and TLR4 in the LPs-mimetic activity of taxol Eur. J. Immunol., 31: 2448-2457.
Corbel, M.J., 1997. Brucellosis: An overview. Emerg. Infect. Dis., 3: 213-221.

De Almeida, L.A., N.B. Carvalho, F.S. Oliveira, T.L. Lacerda and A.C. Vasconcelos et al., 2011. MyD88 and STING signaling pathway are required for IRF3mediated IFN-â induction in response to Brucella abortus infection. PLoS One, 6: e23135-e23135.

Hirsh, D.C., N.J. Maclachlal and R.L. Walker, 2004. Veterinary Microbiology. 2nd Edn., Wiley-Blackwell, USA., ISBN: 9780813803791, pp: 225-228.

Macedo, G.C., D.M. Magnani, N.B. Carvalho, O. BrunaRomero and R.T. Gazzinelli et al., 2008. Central role of MyD88-dependent dendritic cell maturation and proinflammatory cytokine production to control Brucella abortus infection. J. Immunol., 180: 1080-1087.

Sun, D.X. and A.H. Ding, 2006. MyD88-mediated stabilization of interferon-gamma-induced cytokine and chemokine mRNA Nat. Immunol., 7: 375-381.

Takeda, K., T. Kaisho and S. Akira, 2003. Toll-like receptors. Ann. Rev. Immunol., 21: 335-376.

Takeuchi, O., K. Takeda, K. Hoshino, O. Adachi, T. Ogawa and S. Akira, 2000. Cellular responses to bacterial cell wall components are mediated through MyD88-dependent signaling cascades. Int. Immunol., 12: 113-117

Weiss, D.S., K. Takeda, S. Akira, A. Zychlinsky and E. Moreno, 2005. MyD88, but not toll-like receptors 4 and 2, is required for efficient clearance of Brucella abortus. Infect Immun., 73: 5137-51 43.

Yamamoto, M., K. Takeda and S. Akira, 2004. TIR domaincontaining adaptors define the specificity of TLR signaling. Mol. Immunol., 40: 861-868.

Zhang, X.R., W.H. Kuang, Y. Cheng, L. Du and D.L. Zhang et al., 2011. Gene cloning and prokaryotic expression of MyD88 cDNA from buffalo. Genom. Applied Biol., 30: 190-196. 